\title{
Dietary patterns in the healthy oldest old in the healthy aging study and the Canadian longitudinal study of aging: a cohort study
}

\author{
Qianqian Gu', Carly M. Sable ${ }^{1}$, Angela Brooks-Wilson ${ }^{2,3}$ and Rachel A. Murphy ${ }^{1,4^{*}}$
}

\begin{abstract}
Background: Very few people live to eighty-five years and older (the 'oldest old'), and even fewer live to this age without developing chronic diseases. It is important to understand the relationship, if any, of modifiable factors such as diet on healthy aging. However, there are few studies of diet among healthy oldest old, especially in North American populations. We aimed to characterize dietary patterns among 'super-seniors' (SS) within the Canadian Healthy Aging Study.

Methods: 122 SS aged 85 years or older and free of cancer, cardiovascular or pulmonary disease, dementia and diabetes were recruited. Comparisons were made to 12,626 participants aged 65-86 in the Canadian Longitudinal Study of Aging who completed the same 36-item food frequency questionnaire that queried consumption over the prior 12 months of nutrients and foods thought to be important for aging. Dietary patterns were identified with principal component analysis. The odds of being a SS were determined for quartiles of each dietary pattern with logistic regression.
\end{abstract}

Results: Two dietary patterns were identified; a western diet characterized by french fries, red meat, processed meat and a nutrient-rich diet which included fruits, vegetables, whole grains, nuts and seeds among other healthy food choices. Higher scores for both dietary patterns were associated with increased odds of being a SS, however, only the western dietary pattern remained associated with adjustment for covariates (Quartile 4: OR=3.21, 95\% Cl 1.91-5.51).

Conclusions: Our finding adds to the limited evidence on dietary intake among the healthiest oldest old but it is unclear whether assocations reflect generational differences between groups or possible contributions to longevity.

Keywords: Aging, Nutrition, Longevity, Centenarians

\section{Background}

Broadly, healthy aging refers to development and maintenance of the functional ability to enable wellbeing [1]. The emphasis of healthy aging is on healthspan not lifespan with some definitions also emphasizing aging in the absence of disease [2]. The oldest old, those who are

\footnotetext{
* Correspondence: Rachel.murphy@ubc.ca

${ }^{1}$ School of Population and Public Health, University of British Columbia, 2206 East Mall, Vancouver, BC V6T 1Z3, Canada

${ }^{4}$ Cancer Control Research, BC Cancer, Vancouver, BC, Canada

Full list of author information is available at the end of the article
}

eighty-five years and older [3] are among the fastest growing segment of the population in some parts of the world [4]. Few people however, live to this age without developing chronic diseases. It is thus important to understand the influence of modifiable lifestyle factors such as diet on the achievement of exceptional longevity and the role, if any, of these factors on healthspan.

Several studies among long-lived populations have yielded important insight into dietary patterns and nutrients associated with morbidity or life-span. Long-term 
caloric restriction and traditional dietary patterns such as the Okinawan diet and Mediterranean diet have been found to be potentially important for reducing agerelated diseases and supporting longevity [5, 6]. Some studies have compared dietary intake and diet patterns of centenarians to those of 'younger' adult cohorts from the same geographical region [7-12]. Such studies generally report lower energy and/or fat intake for the centenarians as compared to community-dwelling older adults in their $60 \mathrm{~s}$ or $80 \mathrm{~s}$. Conversely, centenarians have also been observed to consume more high-fat foods, and were less likely to avoid dietary cholesterol $[10,13]$.

There are in relative terms, few studies of the oldest old and centenarians in gerontology research, and even fewer studies of populations that have survived to that age in the absence of a major age-related chronic disease. Studies that characterize dietary intake in such populations will add to our knowledge base and provide critical information for hypothesis testing. Our aim was thus to assess the dietary intake of a population of men and women 85 years and older who were free of chronic diseases and compare dietary patterns to adults aged 65 to 86 years using data from a Canada -wide populationbased study of aging. We hypothesized that compared to the younger cohort, older healthy adults would have dietary patterns that more closely follow guidelines for chronic disease risk reduction such as more frequent consumption of fruits and vegetables, whole grains and lean protein.

\section{Methods}

\section{Study participants-healthy aging study}

This study was nested within the Healthy Aging Study [14-17] that was designed to study genetic factors that underlie healthy aging and resistance to age-related diseases. Between 2004 and 2007, participants 85 years and older from Metro Vancouver, British Columbia, Canada were recruited as described in detail previously [17]. Eligibility criteria for super-seniors (SS) included age 85 and older, self-reporting never been diagnosed with or taking medication prescribed for cancer (excluding non-melanoma skin cancer), cardiovascular or pulmonary disease (excluding asthma), dementia or diabetes.

\section{Canadian longitudinal study on aging (CLSA)}

The CLSA is a national longitudinal study of $50,000 \mathrm{Ca}$ nadians aged 45 to 85 years [18]. The Canadian Community Health Survey (CCHS) was used to recruit participants into the CLSA. The sampling frame is nationally representative, although residents in the Northwest Territories, Nunavut, Yukon, some remote regions, persons living on federal First Nations reserves and other First Nations settlements, full-time members of the Canadian Armed Forces and individuals living in institutions were excluded. A subset of 30,000 participants (the "Comprehensive Cohort") underwent in-depth face to face interview questionnaires which included dietary assessment. Baseline data was collected over a 3 year period and completed in June 2015. To provide a large, comparative group for SS's, data was obtained from CLSA participants with dietary information along with covariates that mirrored those collected in our study of SS. Our analysis was confined to CLSA participants aged 65 years and older which provided a comparative group of 12,626 older adults. To most closely approximate the general aging population in Canada, no addition exclusion criteria were applied to the CLSA dataset.

\section{Dietary intake}

Dietary information was collected using the same 36item Short Diet Questionnaire (SDQ) that was developed and used in the CLSA to assess usual consumption frequencies (last 12 months) of key nutrients and foods of importance for health promotion and chronic disease prevention in younger and older adults [19]. The SDQ has been tested for use in community-dwelling older adults (70 years and older) in a subsample of the Québec Longitudinal Study on Nutrition and Successful Aging (NuAge) Study [19]. The SDQ was selected for use in this study to enable direct dietary comparisons with a large national sample of older adults. The SDQ has been validated relative to three 24-h diet recalls and has been shown to be a reasonable approach to obtain usual frequencies of food sources of fats, regular and low-fat food choices, fibre, calcium, vitamin D, whole grains, calciumfortified foods and beverages, and fruits and vegetables [19]. Responses to questions were given as frequency, e.g. number of times food or drink was consumed per day, week or month. Questions about portion size were not included in the SDQ.

\section{Data collection-SS}

Recruitment packages were mailed in January and February 2017 to 177 SS participants who had consented to be re-contacted for further research. Participants were asked to complete a questionnaire on demographics and dietary intake by April 2017. From the potential pool of 177 participants, 2 were not interested in the study, 4 were deceased, 2 had moved, 20 did not respond, and 14 were living in assisted care. Due to the time lapse between recruitment for the Healthy Aging Study and this study, participants were asked whether they had chronic disease: cancer, diabetes, cardiovascular or heart disease, stroke or ischemic attack, peripheral vascular disease, lung disease or emphysema, dementia or Alzheimer's disease, with response options of yes, no, don't know or refused. Participants who answered 'yes' to any of the 
queried incident diseases were excluded $(N=8)$. A further five participants were excluded as they were unable to be subsequently reached to clarify a substantial amount of missing data on the SDQ (32 to 36/36 questions) as well as covariates that would have informed imputation (e.g. sex). The final sample size of SS was 122 . Of the included participants, 2 completed the questionnaire via telephone.

Demographic questions were drawn from the CCHS [20] and included date of birth, sex, marital status, education, ethnicity, income, history of smoking daily, current smoking status, history of alcohol consumption, frequency of alcohol consumption in the prior 12 months, and self-reported height and weight.

\section{Statistical analysis}

Characteristics were compared between groups with two-sided t-tests, chi-square tests or Fisher's exact test. The 36 nutrients and foods were pre-assigned to $21 \mathrm{food}$ groups based on nutrient profiles. Data was cubic root transformed to meet normality. A small proportion of participants had missing dietary data. To maximize our sample size, data were imputed with the mean frequency of intake of the same sex from the same group (SS or CLSA). Principal component analysis was performed to reduce food groups into a smaller set of variables and identify dietary patterns. An orthogonal varimax rotation was used. Scree plots were used to determine the number of factors to retain. A component loading of 0.3 or higher was contained within each factor, forming a dietary pattern. Factor loadings represent the relationship of each food or food group to the underlying factor. Each participant was assigned a factor score for each dietary pattern, and then grouped into quartiles. Participants in quartile 4 of a given diet pattern had the greatest tendency to follow the diet.

Multivariable logistic regression was used to calculate the odds ratios (ORs) and 95\% confidence intervals (CIs) for being a SS. Quartile 1 was the reference for each dietary pattern. Model 1 was unadjusted. The change in coefficient method was used to determine significant confounders. In this procedure, variables were added to the model and remained in the model if they changed the coefficient by at least $10 \%$. Model 2 was adjusted for sex, ethnicity (white, other ethnicity), marital status (living without a partner, living with a partner), household income $(<\$ 50,000, \$ 50,000-\$ 99,999, \geq \$ 100,000)$, education ( $\leq$ high school, $>$ high school but $<$ Bachelor's degree, $\geq$ Bachelor's degree), smoking status (never smoker, former smoker, current smoker), alcohol consumption (never, occasional:<one a month, about once a month, 2-3 times a month, regular: once a week, 2-3 times a week, habitual: 4-5 times a week, or almost every day) and BMI (underweight: $<18.5 \mathrm{~kg} / \mathrm{m}^{2}$, normal weight:
$18.5-24.9 \mathrm{~kg} / \mathrm{m}^{2}$, overweight: $25.0-29.9 \mathrm{~kg} / \mathrm{m}^{2}$, obese: $\geq 30 \mathrm{~kg} / \mathrm{m}^{2}$ ). Models were not adjusted for age as there was minimal overlap between SS and CLSA.

Statistical significance was determined at $\alpha<0.05$. All analyses were performed with $\mathrm{R}$ version 3.4 .3 ( $\mathrm{R}$ Foundation for Statistical Computing, Vienna, Austria).

\section{Results}

Table 1 presents the descriptive statistics of 122 SS and 12,626 CLSA participants. A smaller proportion of SS participants lived with partners (37\% versus $62 \%, p<$ $0.01)$ and had a Bachelor's degree or higher $(37 \%$ versus

Table 1 Characteristics of CLSA participants and super-seniors (SS) in the study sample

\begin{tabular}{|c|c|c|c|}
\hline & $\begin{array}{l}\text { CLSA } \\
\boldsymbol{N}=12,626\end{array}$ & $\begin{array}{l}\text { SS } \\
\boldsymbol{N}=122\end{array}$ & $\boldsymbol{p}$-value \\
\hline Age, Mean (SD) & $73.1(5.7)$ & $90.4(3.6)$ & $<0.001$ \\
\hline Sex, N (\%) & & & 0.08 \\
\hline Male & $6329(50.1)$ & $51(41.8)$ & \\
\hline Female & $6297(49.9)$ & $71(58.2)$ & \\
\hline Ethnicity, N (\%) & & & 0.45 \\
\hline White & $12,191(96.6)$ & $120(98.4)$ & \\
\hline Other ethnicities & $435(3.4)$ & $2(1.6)$ & \\
\hline Marital status, N (\%) & & & $<0.001$ \\
\hline Living without partner & $4759(37.7)$ & $77(63.1)$ & \\
\hline Living with partner & $7866(62.3)$ & $45(36.9)$ & \\
\hline Household income, N (\%) & & & 0.86 \\
\hline$<\$ 50,000$ & $4717(41.0)$ & $50(43.5)$ & \\
\hline$\$ 50,000-99,999$ & 4529 (39.4) & $43(37.4)$ & \\
\hline$\geq \$ 100,000$ & $2257(19.6)$ & $22(19.1)$ & \\
\hline Education, $\mathbf{N}(\%)$ & & & 0.01 \\
\hline$\leq$ high school & $2542(20.2)$ & $35(28.7)$ & \\
\hline$>$ high school, <Bachelor's degree & $3858(30.6)$ & $42(34.4)$ & \\
\hline$\geq$ Bachelor's degree & $6188(49.2)$ & $45(36.9)$ & \\
\hline Smoking, N (\%) & & & 0.001 \\
\hline Never smoker & $5461(43.3)$ & $65(53.3)$ & \\
\hline Former smoker & $6451(51.1)$ & $57(46.7)$ & \\
\hline Current smoker & $713(5.6)$ & $0(0.0)$ & \\
\hline Alcohol (past 12 months), N (\%) & & & $<0.001$ \\
\hline Never & $1640(13.4)$ & $16(13.1)$ & \\
\hline Occasional & $3608(29.4)$ & $29(23.8)$ & \\
\hline Regular & $3250(26.5)$ & 19 (15.6) & \\
\hline Habitual & $3773(30.7)$ & $58(47.5)$ & \\
\hline BMI, N (\%) & & & $<0.001$ \\
\hline Underweight & $106(0.8)$ & $6(5.0)$ & \\
\hline Normal & $3529(28.1)$ & $76(62.8)$ & \\
\hline Overweight & $5388(42.9)$ & $34(28.1)$ & \\
\hline Obese & $3543(28.2)$ & $5(4.1)$ & \\
\hline
\end{tabular}


49\%) compared to CLSA participants. Never smokers were more common among SS (53\% versus $43 \%$ ) and current smokers were less common (0\% versus $5.6 \%$ ) compared to CLSA participants. Differences were also observed for alcohol consumption and BMI, whereby regular drinking and habitual drinking were less and more prevalent in SS than CLSA, respectively. The prevalence of normal BMI (63\% versus 28\%) was higher among SS participants while the prevalence of overweight and obese BMI were lower among SS (overweight: $28 \%$ versus $43 \%$ and obese: $4.1 \%$ versus $28 \%$ ) compared to CLSA participants.

Principal component analysis identified two factors that explained $23 \%$ of the total variance. Foods contained in each factor and factor loadings are shown in Table 2. The first factor was characterized by western foods. The strongest associations for factor 1 were processed meat (factor loading 0.62), red meat (0.59), sauces and gravies (0.58), fried potatoes (0.54) and non-fried potatoes (0.54). Associations between factor 1 and high-

Table 2 Factor loading for the western and nutrient-rich dietary patterns

\begin{tabular}{|c|c|c|}
\hline & Factor 1 & Factor 2 \\
\hline & Loading strength & Loading strength \\
\hline Processed meat & 0.62 & -0.07 \\
\hline Red meat & 0.59 & -0.01 \\
\hline Sauces and gravies & 0.58 & 0.00 \\
\hline Potatoes (fried) ${ }^{1}$ & 0.54 & -0.21 \\
\hline Potatoes (non-fried) ${ }^{2}$ & 0.54 & 0.06 \\
\hline Butter and margarine & 0.46 & -0.03 \\
\hline High sugar snacks ${ }^{3}$ & 0.43 & 0.04 \\
\hline Salty snacks & 0.38 & -0.05 \\
\hline High fat dairy & 0.31 & 0.06 \\
\hline Salad dressing & 0.27 & 0.37 \\
\hline Poultry & 0.25 & 0.25 \\
\hline Fruit juice & 0.24 & 0.02 \\
\hline Eggs & 0.19 & 0.25 \\
\hline Whole grains & 0.03 & 0.46 \\
\hline Low fat dairy & -0.01 & 0.31 \\
\hline Legumes & -0.03 & 0.45 \\
\hline Fish & -0.05 & 0.49 \\
\hline Nuts and seeds & -0.07 & 0.46 \\
\hline Non-starchy vegetables & -0.07 & 0.69 \\
\hline Fruits & -0.19 & 0.61 \\
\hline Calcium-fortified foods ${ }^{4}$ & -0.22 & 0.15 \\
\hline
\end{tabular}

${ }^{1}$ french fries, poutine or other fried potatoes, ${ }^{2}$ boiled, mashed or baked potatoes, ${ }^{3}$ chocolate, cakes, pies, doughnuts, pastries, cookies, muffins, icecream, ice milk, frozen yogurt, milk-based desserts, ${ }^{4}$ calcium fortified foods, calcium fortified juice, calcium fortified milk and other calcium fortified beverages sugar snacks, high-fat dairy, butter and margarine and salty snacks also exceeding factor loading thresholds (> $0.30)$. The second factor was characterized by nutrientrich foods including fruits $(0.61)$, non-starchy vegetables (0.69), whole grains (0.46), legumes (0.45), nuts and seeds (0.46), low-fat dairy products (0.31), salad dressing $(0.37)$ and fish (0.49). The factors are hereafter referred to as 'western dietary factor' and 'nutrient-rich dietary factor'.

Table 3 shows the unadjusted and adjusted ORs and 95\% CIs for being a SS based on logistic regression. The highest quartile of the western dietary factor was associated with greater odds of being a SS (Model 1, Q4 OR = 2.06, 95\% CI 1.29-3.38). After adjustment for confounders, associations were strengthened (Model 2, Q4 adjusted $\mathrm{OR}=3.21,95 \% \mathrm{CI} 1.91-5.51$ ). No associations were observed for quartiles 2 or 3 . The highest quartile of the nutrient-rich dietary factor was associated with greater odds of being a SS in the unadjusted model (Model 1, Q4 OR $=1.75,95 \%$ CI 1.10-2.85). Associations were attenuated in Model 2 after adjustment for covariates $(\mathrm{Q} 4 \mathrm{OR}=1.57,95 \% 0.95-2.66)$. No associations were observed for quartiles 2 or 3 in either model. Variables that were associated with group (SS or CLSA) and dietary pattern included sex, ethnicity, marital status, household income, education, smoking status, alcohol consumption and BMI). Of these, smoking, BMI and alcohol consumption had the largest impact on effect estimates. Covariate estimates are shown in Additional file 1: Table 1.

\section{Discussion}

This study contributes new evidence on dietary patterns and healthy aging in a Canadian population. The

Table 3 Odds ratios (OR) of being a super-senior participant for the western and nutrient-rich dietary factors, results from unadjusted and adjusted logistic regression models among 12,626 CLSA and 122 SS

\begin{tabular}{lllll}
\hline Model 1 & & & Model 2 & \\
\cline { 5 - 6 } \cline { 5 - 6 } OR $(95 \% \mathrm{Cl})$ & p-value & & OR $(95 \% \mathrm{Cl})$ & p-value
\end{tabular}

\begin{tabular}{|c|c|c|c|c|}
\hline \multicolumn{5}{|c|}{ Western dietary factor } \\
\hline Q1 & 1.00 & & 1.00 & \\
\hline Q2 & $0.88(0.49,1.56)$ & 0.66 & $0.99(0.54,1.83)$ & 0.99 \\
\hline Q3 & $0.96(0.54,1.69)$ & 0.89 & $1.24(0.68,2.25)$ & 0.49 \\
\hline Q4 & $2.06(1.29,3.38)$ & 0.003 & $3.21(1.91,5.51)$ & $<0.0$ \\
\hline \multicolumn{5}{|c|}{ Nutrient-rich dietary factor } \\
\hline Q1 & 1.00 & & 1.00 & \\
\hline Q2 & $0.89(0.51,1.54)$ & 0.67 & $0.81(0.45,1.46)$ & 0.49 \\
\hline Q3 & $0.89(0.51,1.54)$ & 0.67 & $0.75(0.41,1.36)$ & 0.34 \\
\hline Q4 & $1.75(1.10,2.85)$ & 0.02 & $1.57(0.95,2.66)$ & 0.09 \\
\hline
\end{tabular}

Model 1 is unadjusted. Model 2 is adjusted for sex, ethnicity, marital status, household income, education, smoking status, alcohol consumption, and BMI. 
nutrient-rich dietary pattern was not associated with being a SS after adjustment for confounders, but the highest quartile of the western dietary pattern was associated with greater odds of being a SS. This was in contrast to our hypothesis as the western dietary pattern was predominately characterized by consumption of foods considered to be less healthy including processed meat, red meat, sauces and gravies, fried and non-fried potatoes, high sugar snacks, salty snacks and high fat dairy products. Nonetheless, it is important to note that the western dietary component contained other foods that did not meet our threshold for component loading (e.g. poultry and eggs) but may have still contributed to associations.

Consistent with findings from other studies of older adults including the Georgia Centenarian Study [21, 22], the Alameda County Study [23] and the Harvard College Alumni Study [23], SS engaged in health behaviors known to promote healthy aging [17]. Compared to CLSA participants, SS had a higher prevalence of never smokers. None were current smokers and few were obese, although habitual alcohol drinking was more prevalent among SS.

Our finding of an apparent disconnect whereby the less healthy western dietary pattern and the nutrientrich dietary pattern were both associated with greater odds of being a SS (in unadjusted models) is similar to findings from the Georgia Centenarian Study [13, 24]. They reported centenarians consumed a more varied diet including higher consumption of milk and grains compared to adults in their 60's. Conversely, centenarians were also more likely to consume whole milk and biscuits and less likely to follow nutrition guidelines for chronic disease risk reduction. The authors suggested that the differences in diet may reflect functional problems in the very old, for example oral health and chewing functions. We did not specifically query difficulty eating but the prevalence was likely low among SS as the inclusion criteria of no chronic diseases correlates with a generally healthy population, and the prevalence of meal replacements was low among SS (data not shown).

It is unclear whether the tendency of SS to follow a western-dietary pattern reflects generational and/or cohort differences with CLSA participants, longevityrelated differences or possibly fatalism (e.g. approaching the end of one's life) which has a negative impact on health behaviors $[25,26]$. For example, higher frequency of high fat foods may reflect ingrained generational dietary patterns. National dietary guidelines were not significantly modified until the 1980's in Canada to emphasize energy balance and moderation (limiting fat, sugar, salt and alcohol) [27]. A prior study by Takeda et al. [8] reported that dietary trends among two cohorts of centenarians studied in 1981 and 1995 differed with respect to cereal, egg, algae products and legume intake that were attributed to generational differences. There is also a potential influence of sociodemographic factors. Education was lower among SS and a higher proportion of SS lived alone, both of which are associated with poor diet [28]. Associations between the western-dietary pattern and SS remained with adjustment for both variables suggesting that marital status and education were not driving the observation.

There are several limitations to our study. While it is not practical to monitor food intake over the lifespan, current diets may not reflect past dietary habits that may be more likely to contribute to healthy aging. Appropriate comparisons for the oldest old are limited. The vast majority of individuals in the same birth cohort as the oldest old generally do not survive to an advanced age and those that do, have chronic disease may be unable to participate in research studies. Our use of the CLSA cohort from the same geographic areas as SS's is the same approach used by centenarian studies $[13,24]$ but it also introduces potential generational and cultural influences. To minimize generational differences we included CLSA participants up to the oldest age range at the time of dietary assessment (age 86), however the majority of CLSA participants were from younger generations than SS; 188 (1.5\%) were aged 85 and older. The SDQ was chosen as the dietary tool in our study to facilitate comparison to the CLSA. However, the SDQ was not designed to quantify the amount of foods consumed or nutrient intake, and is likely prone to recall bias inherent in self-reported assessment of dietary intake. Our results and inferences are thus limited to frequency of consumption rather than amount. This may be an important limitation as total food intake decreases with age [8]. Our sample size of 122 SS although large with respect to the rarity of this population is modest for characterizing dietary patterns, and generalizability to other populations is limited. Lastly, there are numerous factors related to dietary intake and healthy aging that were not measured including social factors, physical activity and genetic variation that would be informative to assess in future studies [29].

\section{Conclusion}

In conclusion, our results suggest a western dietary pattern is associated with increased odds of healthy aging in this sample of older Canadian adults. This finding adds to the limited evidence base of dietary intake among the healthiest oldest old, but its implications should be considered in the context of possible generational differences in diet and limitations of the assessment of food and nutrient frequency but not amount of food.

\section{Supplementary information}

Supplementary information accompanies this paper at https://doi.org/10. 1186/s12877-020-01507-w. 
Additional file 1 Appendix Table 1. Covariate estimates from logistic regression models estimating the odds ratio (OR) of being a super-senior (SS) participant for the western and nutrient-rich dietary factors among $12,626 \mathrm{CLSA}$ and $122 \mathrm{SS}$

\section{Abbreviations}

CCHS: Canadian Community Health Survey; CLSA: Canadian Longitudinal Study on Aging; FFQ: Food frequency questionnaire; OR: Odds ratio; Q: Quartile; SDQ: Short dietary questionnaire; SS: Super seniors

\section{Acknowledgements}

We thank the participants in the Healthy Aging Study and Diane Salema for assistance with project administration.

The CLSA is led by Drs. Parminder Raina, Christina Wolfson and Susan Kirkland. The Healthy Aging Study was initiated with funding from the CIHR.

\section{Authors' contributions}

Conceptualization, RAM, Methodology, RAM, QG, AB-W, Formal Analysis, QG, Writing-Original Draft Preparation, RAM, Writing-Review \& Editing, QG, CS, AB-W, Funding Acquisition, RAM, AB-W. All authors have read and approved the manuscript.

\section{Funding}

Dr. Murphy's time was funded by the Canadian Cancer Society (grant \#704735) and the Michael Smith Foundation for Health Research (grant \#17644). The development, testing and validation of the SDQ were carried out among NuAge study participants as part of the CLSA Phase II validation studies, CIHR 2006-2008. The NuAge study was supported by the Canadian Institutes for Health Research (CIHR), Grant number MOP-62842, and the Quebec Network for Research on Aging, a network funded by the Fonds de Recherche du Québec-Santé. This research was made possible using the data/biospecimens collected by the Canadian Longitudinal Study on Aging (CLSA). Funding for the Canadian Longitudinal Study on Aging (CLSA) is provided by the Government of Canada through the Canadian Institutes of Health Research (CIHR) under grant reference: LSA 9447 and the Canada Foundation for Innovation. This research has been conducted using the CLSA Baseline Tracking Dataset version 3.3, Baseline Comprehensive Dataset version 3.2, under Application Number 171007].

\section{Availability of data and materials}

The SS dataset is available from the corresponding author on reasonable request. The CLSA dataset is available are available from the CLSA but restrictions apply to the availability of these data, which were used under license for the current study and so are not publicly available. Data are however available from the authors upon reasonable request and with permission of the CLSA.

\section{Ethics approval and consent to participate}

The Healthy Aging Study was approved by the joint Clinical Research Ethics Board of BC Cancer and the University of British Columbia, and the Research Ethics Board of Simon Fraser University. The study protocol of the dietary study was approved by the Research Ethics Board of the University of British Columbia. All study participants provided written informed consent.

\section{Consent for publication}

Not applicable.

\section{Competing interests}

The authors declare that they have no conflicts of interest. The opinions expressed in this manuscript are the author's own and do not reflect the views of the Canadian Longitudinal Study on Aging.

\section{Author details}

${ }^{1}$ School of Population and Public Health, University of British Columbia, 2206 East Mall, Vancouver, BC V6T 1Z3, Canada. ${ }^{2}$ Canada's Michael Smith Genome Sciences Centre, BC Cancer, Vancouver, BC, Canada. ${ }^{3}$ Department of Biomedical Physiology and Kinesiology, Simon Fraser University, Burnaby, BC, Canada. ${ }^{4}$ Cancer Control Research, BC Cancer, Vancouver, BC, Canada.
Received: 17 July 2019 Accepted: 6 March 2020

Published online: 16 March 2020

\section{References}

1. What is Healthy Ageing? World Health Organization. https://www.who.int/ ageing/healthy-ageing/en/, Accessed January 9, 2020.

2. Brooks-Wilson AR. Genetics of healthy aging and longevity. Hum Genet 2013;132:1323-38

3. Suzman R, Riley MW. Introducing the "oldest old". Milbank Mem Fund Q Health Soc. 1985;63:177-86.

4. He W Goodkind D, Kowal P. U.S. Census Bureau, International Population Reports, P95/16-1, An Aging World: 2015, U.S. government publishing office, Washington, DC.

5. Willcox BJ, Willcox DC, Todoriki H, Fujiyoshi A, Yano K, He Q, Curb JD, Suzuki M. Caloric restriction, the traditional Okinawan diet, and healthy aging: the diet of the world's longest-lived people and its potential impact on morbidity and life span. Ann N Y Acad Sci. 2007:1114:434-55.

6. Vasto S, Rizzo C, Caruso C. Centenarians and diet: what they eat in the Western part of Sicily. Immun Ageing. 2012;9:10.

7. Arai $Y$, Hirose N, Nakazawa S, Yamamura K, Shimizu K, Takayama M, Ebihara Y, Osono Y, Homma S. Lipoprotein metabolism in Japanese centenarians: effects of apolipoprotein E polymorphism and nutritional status. J Am Geriatr Soc. 2001;49:1434-41.

8. Takeda S, Noji H, Hirose N, Arai Y, Yamamura K, Shimizu K, Homma S, Ebihara Y, Takayama M. Nutritional intake by the oldest elderly Japanese. Tokyo centenarian study 6. Nihon Ronen Igakkai Zasshi. 1998;35:548-58.

9. Chan YC, Suzuki M, Yamamoto S. Dietary, anthropometric, hematological and biochemical assessment of the nutritional status of centenarians and elderly people in Okinawa. Japan J Am Coll Nutr. 1997;16:229-35.

10. Johnson MA, Brown MA, Poon LW, Martin P, Clayton GM. Nutritional patterns of centenarians. Int J Aging Hum Dev. 1992;34:57-76.

11. Houston DK, Johnson MA, Poon LW, Clayton GM. Individual foods and food group patterns of the oldest old. J Nutr Elder. 1994;13:5-23.

12. Fischer JG, Johnson MA, Poon LW, Martin P. Dairy product intake of the oldest old. J Am Diet Assoc. 1995:95:918-21.

13. Poon LW, Jazwinski M, Green RC, Woodard JL, Martin P, Rodgers WL, Johnson MA, Hausman D, Arnold J, Davey A, Batzer MA, Markesbery WR, Gearing M, Siegler IC, Reynolds S, Dai J. Methodological considerations in studying centenarians: lessons learned from the Georgia centenarian studies. Annu Rev Gerontol Geriatr. 2007;27:231-64.

14. Halaschek-Wiener J, Amirabbasi-Beik M, Monfared N, Pieczyk M, Sailer C, Kollar A, Thomas R, Agalaridis G, Yamada S, Oliveira L, Collins JA, Meneilly G, Marra MA, Madden KM, Le ND, Connors JM, Brooks-Wilson AR. Genetic variation in healthy oldest-old. PLoS One. 2009;4:e6641.

15. Halaschek-Wiener J, Vulto I, Fornika D, Collins J, Connors JM, Le ND, Lansdorp PM, Brooks-Wilson A. Reduced telomere length variation in healthy oldest old. Mech Ageing Dev. 2008;129:638-41.

16. Tindale LC, Leach S, Ushey K, Daley D, Brooks-Wilson AR. Rare and common variants in the Apolipoprotein $\mathrm{E}$ gene in healthy oldest old. Neurobiol Aging. 2014;35:727 e721-3.

17. Halaschek-Wiener J, Tindale LC, Collins JA, Leach S, McManus B, Madden K, Meneilly G, Le ND, Connors JM, Brooks-Wilson AR. The super-seniors study: phenotypic characterization of a healthy $85+$ population. PLoS One. 2018; 13:e0197578.

18. Raina PS, Wolfson C, Kirkland SA, Griffith LE, Oremus M, Patterson C, Tuokko $\mathrm{H}$, Penning M, Balion CM, Hogan D, Wister A, Payette H, Shannon H, Brazil K. The Canadian longitudinal study on aging (CLSA). Can J Aging. 2009;28: 221-9.

19. Shatenstein $B$, Payette $H$. Evaluation of the relative validity of the short diet questionnaire for assessing usual consumption frequencies of selected nutrients and foods. Nutrients. 2015:7:6362-74.

20. Canadian Community Health Survey - Annual Component (CCHS). Statistics Canada.

21. Johnson MA, Davey A, Hausman DB, Park S, Poon LW, Georgia CS. Dietary differences between centenarians residing in communities and in skilled nursing facilities: the Georgia Centenarian Study. Age (Dordr). 2006;28:33341.

22. Nickols-Richardson SM, Johnson MA, Poon LW, Martin P. Demographic predictors of nutritional risk in elderly persons. J Appl Gerontol. 1996;15: 361-75. 
23. Breslow L, Breslow N. Health practices and disability: some evidence from Alameda County. Prev Med. 1993;22:86-95.

24. Nickols-Richardson SM, Johnson MA, Poon LW, Martin P. Mental health and number of illnesses are predictors of nutritional risk in elderly persons. Exp Aging Res. 1996;22:141-54.

25. Espinosa de Los Monteros K, Gallo LC. The relevance of fatalism in the study of Latinas' cancer screening behavior: a systematic review of the literature. Int J Behav Med. 2011;18:310-8.

26. Shen L, Condit CM, Wright L. The psychometric property and validation of a fatalism scale. Psychol Health. 2009;24:597-613.

27. History of Canada's Food Guides from 1942 to 2007. Government of Canada; 2007.

28. Ball K, Crawford D, Mishra G. Socio-economic inequalities in women's fruit and vegetable intakes: a multilevel study of individual, social and environmental mediators. Public Health Nutr. 2006:9:623-30.

29. Paffenbarger RS Jr, Kampert JB, Lee IM, Hyde RT, Leung RW, Wing AL. Changes in physical activity and other lifeway patterns influencing longevity. Med Sci Sports Exerc. 1994;26:857-65.

\section{Publisher's Note}

Springer Nature remains neutral with regard to jurisdictional claims in published maps and institutional affiliations.

Ready to submit your research? Choose BMC and benefit from:

- fast, convenient online submission

- thorough peer review by experienced researchers in your field

- rapid publication on acceptance

- support for research data, including large and complex data types

- gold Open Access which fosters wider collaboration and increased citations

- maximum visibility for your research: over $100 \mathrm{M}$ website views per year

At BMC, research is always in progress.

Learn more biomedcentral.com/submissions 